\title{
Infrared and Ultraviolet Observations of VIRGOHI 21 and NGC 4254's Outer Disk
}

\author{
Zhong Wang*, Stephanie Bush ${ }^{\dagger}$, Doug McElroy** and Robert Minchin ${ }^{*}$ \\ *Smithsonian Astrophysical Observatory, Cambridge, MA 02138 \\ ${ }^{\dagger}$ Harvard-Smithsonian Center for Astrophysics, 60 Garden Street, Cambridge, MA 02138 \\ ${ }^{*}$ Spitzer Science Center, California Institute of Technology, Pasadena, CA 91125 \\ ${ }_{\ddagger}$ Arecibo Observatory, HC03 Box 53995, Arecibo, PR 00612
}

\begin{abstract}
We present the results of Spitzer and Galex observations of gas/dust and star formation activities in the extreme outer disk of Virgo galaxy NGC 4254 and its surrounding regions. These observations were motivated in part by the potential existence of a "dark galaxy" in the vicinity. In the intergalactic VIRGOHI 21 region where the free-floating HI gas is found, neither UV nor mid-IR shows corresponding emission, thus providing stringent upper limits on the stellar mass and star formation rate in these clouds.

On the other hand, we find clearly discernible excess ultraviolet emission in parts of the extended disk of NGC 4254, which is yet unseen in the optical and infrared. These UV emission appears different from the so-called "XUV disks" of other nearby galaxies in both their distribution pattern and physical origin, which we suggest is directly related to the gas concentration of VIRGOHI 21.
\end{abstract}

Keywords: XUV Disk, Galaxy interaction, Galex, Spitzer, HI

PACS: $95.85 . \mathrm{Hp}, 95.85 . \mathrm{Mt}, 98.58 . \mathrm{Nk}$

\section{INTRODUCTION}

As large extragalactic neutral hydrogen surveys improve in both sensitivity and sky coverage, more detailed studies of individual HI clouds have also been carried out [e.g. $1,2,3,4]$. In particular, the possible existence of "(optically) dark" galaxies, i.e. those of very high mass-to-light ratios as inferred from comparing with deep optical data, has received much attention. A case in point is the VIRGOHI 21 region studied by Minchin et al. [5, 6] based on their VIRGOHI survey [1]. Located on the edge of the Virgo cluster, this region appears to have one or more large HI clouds with no detectable optical emission. More recently, however, deeper Arecibo observations [7] point to a tidal origin of the HI mass. Indeed, newer models $[8,9]$ make a intriguing case that a history of galaxy-galaxy interaction involving the giant nearby Virgo galaxy NGC 4254 (M99) could have produced the observed HI distribution and kinematics.

\section{OBSERVATIONS}

The region around VIRGOHI 21 was mapped with both IRAC and MIPS as part of the Spitzer Cycle 4 GTO observations. The areas covered by the two instruments are approximately $10^{\prime} \times 25^{\prime}$ and $15^{\prime} \times 35^{\prime}$, respectively. The total integration times are approximately $300 \mathrm{~s}$ for IRAC (all four bands), and 200s for MIPS (24 and $70 \mu \mathrm{m}$ ). The outer disk of NGC 4254 has been covered by the Spitzer Legacy program SINGS 
[10], and the data were made available through the Spitzer data archive at the SSC. The IRAC exposures are $120 \mathrm{~s}$ at each position on average.

We observed the VIRGOHI 21 and its surrounding regions with Galex in both FUV and NUV bands. The Galex field of view is large enough to also cover the entire disk of NGC 4254. NGC 4254 was also observed in a Galex GTO survey program [11], but our $\mathrm{GO}$ observations were about four times deeper in exposure time and thus much more sensitive to the faint, diffuse UV emissions.

\section{COMPARISON WITH HI AND OTHER DATA}

In the mid-infrared data, numerous stellar and extragalactic sources seen in the deep optical data can be identified in the IRAC 3.6 and $4.5 \mu \mathrm{m}$ bands. Some point sources are also found in the 5.8 and $8.0 \mu \mathrm{m}$ bands, as are a few in the MIPS images. However, there is NO obvious counterpart of the HI cloud in the infrared images, especially for non-point sources as it might have be expected. In particular, comparing the 5.8 and $8.0 \mu \mathrm{m}$ images which are sensitive to warm dust and PAH emissions with those of shorter wavelengths suggests very low level of dust with respect to the measured column density of neutral hydrogen gas. The upper limit to the implied gas-to-dust ratio is at least 3 times those of typical values measured in the outer disks of gas-rich galaxies.

We also do not find apparent excess UV at the position of the VIRGOHI 21 cloud. Because the local dust content is relatively low, ultraviolet emission should have been detected if there was either low-level, on-going star formation, or a young stellar population. The lack of detectable UV emission, in combination with flux limits in the optical and infrared, suggests that the HI gas concentration is probably not self-gravitating.

On the other hand, we find wide-spread NUV (2310 $\AA$ ) and FUV (1550 $\AA)$ emissions in the extreme outer disk of NGC 4254 (Figure 1). More specifically, the UV emission appear to concentrate in two regions, i.e. the SW and NE quadrants of the galaxy. These regions have a patchy appearance, similar to those found in the outer disk of M83 $[12,13]$. Each bright ' $k$ not' is clearly more extended than a typical point source $\left(5^{\prime \prime}\right.$ FWHM). The overall distribution of the UV knots is not random, neither can they be traced as direct extensions of the galaxy's spiral structure. Instead, comparison with the newer Arecibo HI map of the area [7] shows that these are spatially associated with the "debris" HI clouds linking the outer disk of NGC 4254 to VIRGOHI 21, spanning a distance of more than 300 kpcs.

\section{GALAXY INTERACTION OR RAM PRESSURE STRIPPING?}

As pointed out by Thilker et al [14], with Galex imaging many nearby galaxies show extended disk (a.k.a. the "XUV disk") in the UV far beyond the conventional disk radius. But these same authors find that the majority of these XUV disks also have an extended HI disk, possibly due to infall or accretion. The scenario of inside-out disk formation, where the outermost part of the disk is experiencing more recent star formation has been suggested [15]. However, the situation in the outer disk of NGC 4254 is somewhat different in two main aspects: First, the distribution of UV emission in the outer disk is 

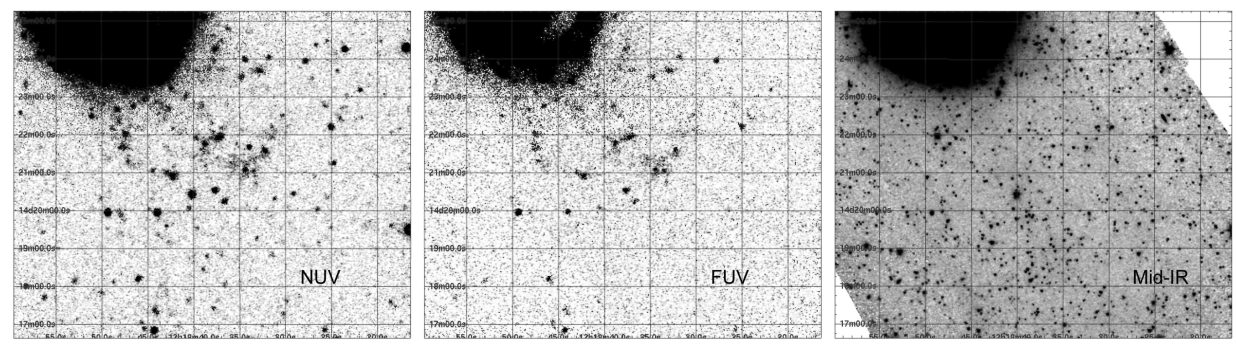

FIGURE 1. NUV, FUV (Galex) and Mid-IR (Spitzer $3.6 \mu \mathrm{m}$ ) images of the SW quadrant of NGC 4254 .

far from symmetric; Secondly, the HI found in nearby regions individually have large offsets from the locations of UV-emitting knots.

In light of the dynamical simulations of Vollmer et al. [16] and Duc et al. [8,9], we attribute the UV emission to star formation induced by some disturbance either because of the ram pressure effect due to NGC 4254's motion towards the Virgo center, or a close encounter with a fly-by galaxy. At this point we tentatively prefer the former model mainly based on time scale argument: the spectral energy distribution of the UV emission suggests a relatively young star forming history (Wang et al., in prep.) The tidal effect, in comparison, may take too long a time to account for the spatial separation between the UV emitting region in the outer disk and the main HI concentration in the VIRGOHI 21 cloud.

\section{REFERENCES}

1. J. I. Davies et al., MNRAS 349, 922-932 (2004).

2. E. Brinks, F. Walter, \& E. D. Skillman, in Dark Galaxies and Lost Baryons, eds. J. I. Davies and M. J. Disney, IAU Symp. 244, CUP, Cambridge, 120-126 (2007).

3. A. Chung, J. H. van Gorkom, J. D. P. Kenney, \& B.Vollmer, ApJ 659, L115-119 (2007).

4. R. Giovanelli et al., AJ 133, 2569-2583 (2007).

5. R. Minchin et al., ApJ 622, L21-24 (2005).

6. R. Minchin et al., ApJ 670, 1056-1064 (2007).

7. M. P. Haynes, R. Giovanelli, \& B. Kent, ApJ 665, L19-22 (2007).

8. A.-P. Duc, F. Bournaud, \& E. Brinks, in Dark Galaxies and Lost Baryons, eds. J. I. Davies and M. J. Disney, IAU Symp. 244, CUP, Cambridge, 216-225 (2007).

9. A.-P. Duc, \& F. Bournaud, ApJ 673, 787-797 (2008).

10. R. C. Kennicutt, Jr., et al., PASP 115, 928-952 (2003).

11. A. Gil de Paz et al., ApJS 173, 185-255 (2007).

12. D. A. Thilker et al., ApJ 619, L79-82 (2005).

13. A. Gil de Paz et al., ApJ 661, 115-134 (2007).

14. D. A. Thilker et al., ApJS 173, 572-596 (2007).

15. S. Boissier et al., ApJS 173, 524-537 (2007).

16. B. Vollmer, W. Huchtmeier, \& W. van Driel, A\&A 439, 921-933 (2005). 